\title{
EARLY OPTICAL BRIGHTENING IN GRB 071010B
}

\author{
J. H. Wang, ${ }^{1}$ M. E. Schwamb ${ }^{2}$ K. Y. Huang, ${ }^{1}$ C. Y. Wen, ${ }^{1}$ Z. W. Zhang, ${ }^{3}$ S. Y. Wang, ${ }^{1}$ W. P. Chen, ${ }^{3}$ F. B. Bianco, ${ }^{4}$ \\ R. Dave, ${ }^{5}$ M. J. Lehner, ${ }^{1,4}$ S. L. Marshall, ${ }^{6,7}$ R. Porrata, ${ }^{8}$ C. Alcock, ${ }^{5}$ Y. I. Byun, ${ }^{9}$ K. H. Cook, ${ }^{7}$ \\ S. K. KING, ${ }^{1}$ T. LeE, ${ }^{1}$ AND Y. URATA ${ }^{1,10}$ \\ Received 2008 March 7; accepted 2008 April 7; published 2008 April 24
}

\begin{abstract}
We report the detection of early (60-230 s) optical emission of the gamma-ray burst afterglow of GRB 071010B. No significant correlation with the prompt $\gamma$-ray emission was found. Our high time resolution data combining with other measurements within 2 days after the burst indicate that GRB 071010B is composed of a weak early brightening $(\alpha \sim 0.6)$, probably caused by the peak frequency passing through the optical wavelengths, followed by a decay $(\alpha \sim-0.51)$, attributed to continuous energy injection by patchy jets.
\end{abstract}

Subject headings: gamma rays: bursts - gamma rays: observations

Online material: color figures

\section{INTRODUCTION}

With the launch of the Swift $\gamma$-ray explorer (Gehrels et al. 2004) in late 2004, great progress has been made in the study of early optical afterglow of gamma-ray bursts (GRBs). Two broad classes have been proposed (Vestrand et al. 2006) for the optical emission detected during the first few minutes after a GRB: (1) prompt optical emission correlated in time with the prompt $\gamma$-ray emission (e.g., GRB 041219 and GRB 050820a; Vestrand et al. 2005, 2006); (2) early optical afterglow after the $\gamma$-ray emission (e.g., GRB 990123 and GRB 050401; Akerlof et al. 1999; Rykoff et al. 2005).

The standard relativistic blast wave ("fireball") model (e.g., Sari \& Piran 1997a; Meśzaŕos 2002) attributes the prompt $\gamma$ ray emission to the internal shocks in the ultrarelativistic jet generated by the central engine (Narayan et al. 1992; Rees \& Meśzaŕos 1994; Sari \& Piran 1997b). The prompt optical emission is known to vary simultaneously with the prompt $\gamma$-ray emission, indicative of their common origin. The early optical afterglow, on the other hand, is considered to arise from interaction between the internal shocks and the surrounding medium (so-called "external shocks"; Rees \& Meśzaŕos 1992; Meśzaŕos \& Rees 1993; Katz 1994; Sari \& Piran 1995). The early optical afterglow may start during the prompt $\gamma$-ray emission, and persist for 10 minutes or longer after the prompt $\gamma$ ray emission has faded. So far, only GRB 050820a has been observed to show a temporal correlation between the two dif-

\footnotetext{
${ }^{1}$ Institute of Astronomy and Astrophysics, Academia Sinica, P.O. Box 23141, Taipei 106, Taiwan.

${ }^{2}$ Division of Geological and Planetary Sciences, California Institute of Technology, 1201 East California Boulevard, Pasadena, CA 91125.

${ }^{3}$ Institute of Astronomy, National Central University, Chung-Li 32054, Taiwan.

${ }^{4}$ Harvard-Smithsonian Center for Astrophysics, 60 Garden Street, Cambridge, MA 02138.

${ }^{5}$ Initiative in Innovative Computing, Harvard University, 60 Oxford Street, Cambridge, MA 02138.

${ }^{6}$ Kavli Institute of Particle Astrophysics and Cosmology, 2575 Sand Hill Road, MS 29, Menlo Park, CA 94025.

${ }^{7}$ Institute of Geophysics and Planetary Physics, Lawrence Livermore National Laboratory, Livermore, CA 94550.

${ }^{8}$ Department of Physics, University of California, Berkeley, CA 94270.

${ }^{9}$ Department of Astronomy, Yonsei University, 134 Shinchon, Seoul 120749, Korea.

${ }^{10}$ Department of Physics, Saitama University, Shimookubo, Saitama 3388570 Japan.
}

ferent optical components. Such a correlation suggests that the early afterglow is associated with the impulsive energy released by the prompt emission, which is consistent with what is expected in the fireball model for an external forward shock. Fast photometry in the early phase of emission is therefore important to reveal the two optical components and to study the interaction of the GRB ejecta with the surrounding medium.

In this Letter, we report on our high time resolution detection of the optical emission of GRB 071010B in the first few minutes with the TAOS (Taiwanese-American Occultation Survey) telescopes (Alcock et al. 2003; Chen et al. 2007).

\section{THE TAOS OBSERVATION AND RESULTS}

The TAOS system consists of an array of four robotic telescopes, TAOS-A, TAOS-B, TAOS-C, and TAOS-D, with separations ranging from 6 to $65 \mathrm{~m}$. Each telescope has a $50 \mathrm{~cm}$ aperture imaging to a corrected Cassegrain focus at $\mathrm{f} / 1.9$ with a 5000-7200 $\AA$ passband. Each telescope is equipped with a Spectral Instruments Series 800 CCD camera with a $2048 \times$ 2052 E2V CCD 42-40 chip with two readout channels. The optical system gives a $3 \mathrm{deg}^{2}$ field of view. More details about the TAOS telescope system are described by Lehner et al. (2008). The main scientific goal of the TAOS project is to detect and characterize the small (a few km across) objects beyond Neptune, but the robotic system is well suited to respond efficiently to GRBs.

Upon arrival of a GCN alert, the TAOS scheduler daemon makes the decision whether to interrupt the regular observing session to follow up on the GRB, based on the observability of the target, duplicate packets, alert retracted by the GCN, etc. Currently the TAOS system responds to, weather permitting, any GRB above $25^{\circ}$ elevation, within 2 hours of the burst. The advantage of the TAOS system for the GRB studies is its capability to respond rapidly to an alert with fast photometry. This allows us to study early optical behavior of a GRB. Currently our system can point to the burst coordinates provided by Swift in less than $30 \mathrm{~s}$ and start observing. A GRB alert is followed up for 30 minutes, with each TAOS telescope taking a different exposure time, i.e., with different cadences between telescopes, from a fraction of a second to tens of seconds. Targets brighter than $R \sim 18$ mag can be readily detected. This setup of multiple telescopes taking different exposure times maximizes the chance to detect various types of GRB optical 


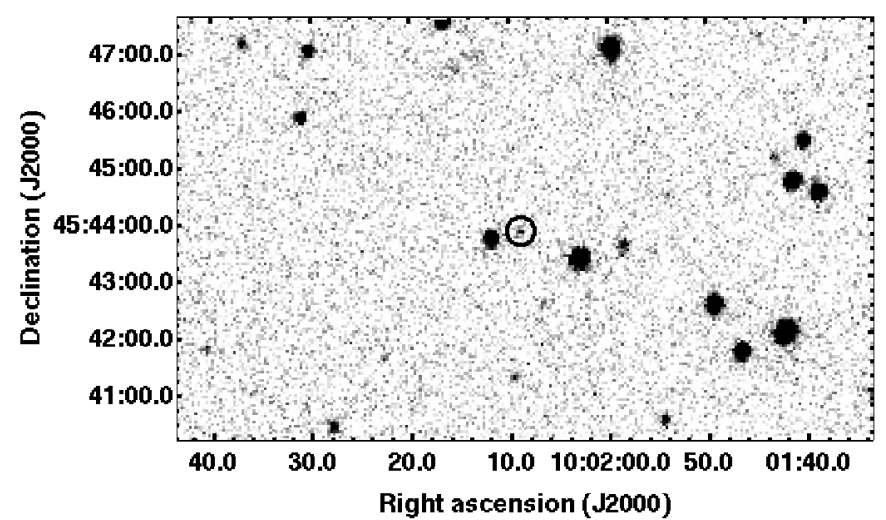

FIG. 1.-One of the TAOS afterglow images taken with the TAOS-B telescope at $60 \mathrm{~s}$ after the burst of GRB 071010B. The integration time was $5 \mathrm{~s}$.

counterparts and enables cross-checking of photometric reliability.

On 2007 October 10, the Swift Burst Alert Telescope (BAT) detected GRB 071010B at 20:45:47 UT. This burst showed a main FRED (fast rise-exponential decay) pulse lasting $\sim 20 \mathrm{~s}$, with a precursor some $45 \mathrm{~s}$ before the main pulse, an extended tail emission, and a third, weak peak around $95 \mathrm{~s}$ after the burst (Markwardt et al. 2007). The $T_{90}$ duration of this event is 35.74 $\pm 0.5 \mathrm{~s}$ in the 15-350 keV range (Markwardt et al. 2007). Suzaku WAM also detected the main spike (Kira et al. 2007; Y. Urata et al. 2008, in preparation).

A bright X-ray afterglow was detected by the Swift X-Ray Telescope (XRT) $6800 \mathrm{~s}$ after the burst (Kennea et al. 2007). In addition, a bright optical afterglow was detected by several ground-based telescopes (Oksanen 2007; Kann et al. 2007a; Im et al. 2007a; Kocevski et al. 2007; Xin 2007; Klunko et al. 2007; Rumyantsev \& Pozanenko 2007). The spectral measurements of the afterglow by the Gemini North telescope (Cenko et al. 2007) and Keck telescope (Stern et al. 2007) indicate a redshift of $z=0.947$ for this event. A jet break was also detected with late-time observations (Kann et al. 2007c; Im et al. 2007a; Y. Urata et al. 2008, in preparation).

The TAOS telescopes began to observe GRB 071010B about $38 \mathrm{~s}$ after the GCN alert-or $52 \mathrm{~s}$ after the burst. A sequence of 1,5 , and 25 s exposures were taken, respectively, by TAOSA, TAOS-B, and TAOS-D. The sky conditions were favorable but observations were terminated after 3 minutes because of the twilight shutdown set by the scheduler. Such a constraint has since been lifted to allow completion (30 minutes) of a GRB follow-up, with the fourth TAOS telescope now designated to take $0.25 \mathrm{~s}$ data.

The images were processed by a standard routine including bias subtraction and flat-fielding using IRAF. Detection was clearly seen in the individual images of TAOS-B and TAOS$\mathrm{D}$, but not in TAOS-A. The images of TAOS-A were co-added to increase the signal-to-noise ratio in the analysis. The DAOPHOT package (Stetson 1987) was then used to perform pointspread function (PSF) photometry. In each image, six stars around the GRB position were used to create the PSF model. For photometric calibration, we used nine reference stars $(0.29<V-R<0.61)$ in the GRB field given by Henden (2007). Our photometric results, including those of individual images by TAOS-B (Fig. 1) and TAOS-D, and of stacked images by TAOS-A, are listed in Table 1 . Both the photometric and systematic calibration errors were included in the magnitude error. A similar result was obtained with differential pho-
TABLE 1

Log of GRB 071010B Optical Afterglow ObSERVATions

\begin{tabular}{|c|c|c|c|c|}
\hline$t_{\text {start }}$ & $t_{\text {end }}$ & $\begin{array}{c}\text { Exposure } \\
\text { (s) }\end{array}$ & Magnitude & Telescope \\
\hline 60.28 & 65.28 & 5 & $16.88 \pm 0.15$ & TAOS-B \\
\hline 64.87 & 145.19 & $1 \mathrm{~s} \times 20$ & $16.64 \pm 0.19$ & TAOS-A \\
\hline 68.48 & 73.48 & 5 & $16.84 \pm 0.19$ & TAOS-B \\
\hline 76.78 & 81.78 & 5 & $17.01 \pm 0.19$ & TAOS-B \\
\hline $80.38 \ldots \ldots$ & 105.38 & 25 & $16.66 \pm 0.05$ & TAOS-D \\
\hline 84.98 & 89.98 & 5 & $16.95 \pm 0.19$ & TAOS-B \\
\hline $93.28 \ldots \ldots$ & 98.28 & 5 & $16.74 \pm 0.15$ & TAOS-B \\
\hline $101.59 \ldots \ldots$ & 106.59 & 5 & $16.70 \pm 0.14$ & TAOS-B \\
\hline $108.59 \ldots \ldots$ & 133.59 & 25 & $16.59 \pm 0.06$ & TAOS-D \\
\hline 109.79 & 114.79 & 5 & $16.63 \pm 0.13$ & TAOS-B \\
\hline 118.09 & 123.09 & 5 & $16.60 \pm 0.13$ & TAOS-B \\
\hline 126.29 & 131.29 & 5 & $16.42 \pm 0.12$ & TAOS-B \\
\hline 134.49 & 139.49 & 5 & $16.41 \pm 0.11$ & TAOS-B \\
\hline $136.90 \ldots \ldots$ & 161.90 & 25 & $16.52 \pm 0.06$ & TAOS-D \\
\hline $142.80 \ldots \ldots$ & 147.80 & 5 & $16.51 \pm 0.09$ & TAOS-B \\
\hline $149.49 \ldots \ldots$ & 226.41 & $1 \mathrm{~s} \times 19$ & $16.55 \pm 0.18$ & TAOS-A \\
\hline 151.10 & 156.10 & 5 & $16.67 \pm 0.12$ & TAOS-B \\
\hline $159.30 \ldots \ldots$ & 164.30 & 5 & $16.53 \pm 0.12$ & TAOS-B \\
\hline 165.20 & 190.20 & 25 & $16.68 \pm 0.07$ & TAOS-D \\
\hline $167.50 \ldots \ldots$ & 172.50 & 5 & $16.50 \pm 0.12$ & TAOS-B \\
\hline $175.80 \ldots \ldots$ & 180.80 & 5 & $16.86 \pm 0.20$ & TAOS-B \\
\hline $184.00 \ldots \ldots$ & 189.00 & 5 & $16.59 \pm 0.14$ & TAOS-B \\
\hline $192.20 \ldots \ldots$ & 197.20 & 5 & $16.48 \pm 0.13$ & TAOS-B \\
\hline $193.51 \ldots \ldots$ & 218.51 & 25 & $16.68 \pm 0.08$ & TAOS-D \\
\hline $200.41 \ldots \ldots$ & 205.41 & 5 & $16.67 \pm 0.14$ & TAOS-B \\
\hline $208.61 \ldots \ldots$ & 213.61 & 5 & $16.65 \pm 0.12$ & TAOS-B \\
\hline $216.91 \ldots \ldots$ & 221.91 & 5 & $16.88 \pm 0.17$ & TAOS-B \\
\hline $225.11 \ldots \ldots$ & 230.11 & 5 & $16.57 \pm 0.15$ & TAOS-B \\
\hline
\end{tabular}

tometry by the SExtractor software package 2.3 (Bertin \& Arnouts 1996) using a nearby reference star (USNOB1 1355.0217787, R.A. $=10^{\mathrm{h}} 02^{\mathrm{m}} 23.577^{\mathrm{s}}, \quad$ decl. $=$ $\left.+45^{\circ} 34^{\prime} 54.38^{\prime \prime}, R 2=15.12 \mathrm{mag}\right)$. The detection threshold was that an object must have at least 3-10 connected pixels and a flux in excess of 1.5 times the local background noise. The MAGAPER estimator in SExtractor was used to determine the magnitude of the source. The TAOS camera employs a broad, nonstandard filer, with an effective wavelength close to standard $R$ (Lehner et al. 2008). Zero point transformation to standard $R$ can be effected with a deviation of $\sim 0.007$ to the result in Henden (2007).

Figure 2 shows the optical light curves of GRB 071010B taken by the three TAOS telescopes during 60-230 s after the burst. Our data from three telescopes showed consistently a mild increase in flux, with a best-fit power-law index $\alpha=$ $0.10 \pm 0.07\left(\chi^{2} / \nu=1.15\right.$ for $\left.\nu=26\right)$, defined by $F(t) \propto t^{\alpha}$, where $F$ is the flux at time $t$ after the burst. Following this mild brightening, GRB 071010B appeared to show a shallow decay ( $\alpha \sim-0.48$ ) during 1020-21,600 s after the burst (Templeton et al. 2007). To combine our TAOS measurements with GCN reports covering up to 2 days after the burst (Oksanen 2007; Kann et al. 2007b; Kocevski et al. 2007; Im et al. 2007b; Klunko et al. 2007; Antonyuk et al. 2007), we recalibrated all the data using the reference stars in Henden (2007) in the GRB 071010B field. The data of Oksanen (2007) and Im et al. (2007b) were calibrated by USNO B1.0 stars with red magnitude; the USNO B1.0 values are on average 0.09 mag fainter than those reported by Henden. The reference star (R.A. = $10^{\mathrm{h}} 02^{\mathrm{m}} 09.93^{\mathrm{s}}$, decl. $\left.=+45^{\circ} 41^{\prime} 41.1^{\prime \prime}\right)$ reported by Kann et al. (2007b), Klunko et al. (2007), and Antonyuk et al. (2007) is 0.227 mag fainter than that given by Henden. The entire, rescaled light curve, covering from 1 minute to almost 2 days after the burst, was then fitted with a broken power-law function, $F(\nu, t)=F_{\nu}^{*} /\left[\left(t / t_{\mathrm{b}}\right)^{-\alpha_{1}}+\left(t / t_{\mathrm{b}}\right)^{-\alpha_{2}}\right]$, where $t_{b}$ is the break 


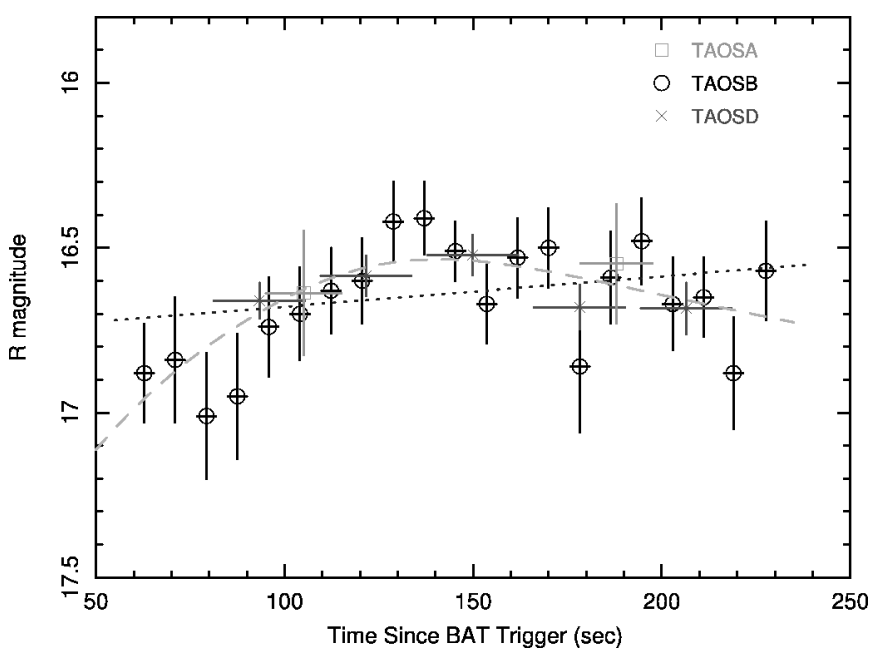

FIG. 2.-Early optical light curves of GRB 071010B taken with TAOS-A (squares; stacked images), TAOS-B (circles), and TAOS-D (asterisks). The linear least-squares fit (dotted line) to all TAOS data shows a mild increase of flux. The dashed line is the best broken power-law fit with other measurements as shown in Fig. 3. [See the electronic edition of the Journal for a color version of this figure.]

time, $\alpha_{1}$ and $\alpha_{2}$ are the power-law indices before and after $t_{b}$, and $F_{v}^{*}$ is the flux at $t_{b}$ (Beuermann et al. 1999). Figure 3 shows the TAOS and recalibrated measurements, together with the best-fit broken power-law function. It is seen that the light curve has an initially rising phase $\left(\alpha_{1}=0.62 \pm 0.34\right)$ followed by a decay $\left(\alpha_{2}=-0.51 \pm 0.01\right)$ after the break time $t_{b}=$ $137.0 \pm 19.0 \mathrm{~s}\left(\chi^{2} / \nu=0.98\right.$ for $\left.\nu=31\right)$. For clearance, the best broken power-law fit is also shown in Figure 2 with TAOS data only.

\section{DISCUSSION AND CONCLUSIONS}

The TAOS light curve was compared with the $\gamma$-ray observations for possible temporal correlation. The preliminary highlevel BAT data (15-350 keV), downloaded from the Swift Data Center (SDC), ${ }^{11}$ were binned to $5 \mathrm{~s}$ in order to compare with the TAOS-B $5 \mathrm{~s}$ data set (Fig. 4). The optical and $\gamma$-ray light curves appear quantitatively similar. In particular the optical brightening during 80-140 s after the burst seems to correspond to the weak, third peak at $95 \mathrm{~s}$ in the BAT light curve reported by Markwardt et al. (2007). The $\gamma$-ray signal was, however, already too weak in this time interval for the optical emission to be the counterpart of the prompt $\gamma$-ray emission.

The early optical emission of GRB 071010B, with a brightening followed by a shallow decay, is unusual. The initial deceleration of the fireball by the reverse shock would produce a bright peak, such as observed in the well-known GRB 990123, which exhibited a sharp brightening of $\sim 3$ mag within $25 \mathrm{~s}$, until $50 \mathrm{~s}$ after the burst (Akerlof et al. 1999). The reverse shock is short-lived and subsequent cooling results in a fast decay, e.g., $\alpha_{2} \sim-2.5$ for GRB 990123 (Akerlof et al. 1999). In comparison, GRB $071010 \mathrm{~B}$ brightened only $0.6 \mathrm{mag}$ in 80 $\mathrm{s}$, and had a later break time $\left(t_{b} \sim 140 \mathrm{~s}\right)$ plus a much shallower decay $\left(\alpha_{2} \sim-0.51\right)$. The reverse shock therefore should not be responsible for the optical emission that we detected.

Electrons accelerated in the shock emit synchrotron radiation. As the fireball slows down, the synchrotron peak frequency moves progressively to a lower frequency. The optical

\footnotetext{
${ }^{11}$ See http://heasarc.gsfc.nasa.gov/docs/swift/sdc/data_products.html.
}

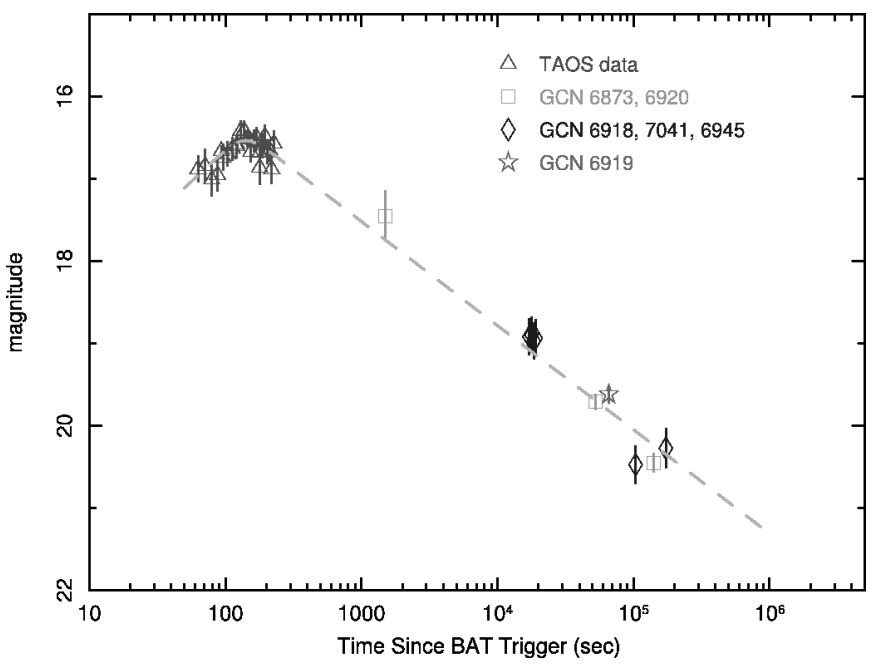

FIG. 3.-Optical light curve of GRB 071010B. The dashed line indicates the best fit by the broken power-law function to the TAOS observations in the first 3 minutes (this work) and data from the literature up to 2 days after the burst (Oksanen 2007; Kann et al. 2007b; Kocevski et al. 2007; Im et al. 2007b; Klunko et al. 2007; Antonyuk et al. 2007). [See the electronic edition of the Journal for a color version of this figure.]

weak brightening that we detected could arise when the synchrotron peak frequency passed through the optical wavelengths. The optical light curve of the forward-shock emission is expected to show an initial rising $\left(t^{0.5}\right)$, followed by a normal decay $t^{3(1-p) / 4}$, where $p$ is the power-law index of the electron spectrum with $p>2$ (e.g., GRB 041006; Urata et al. 2007). The optical light curve of GRB 071010B has a rising rate $\left(\alpha_{1}=0.62 \pm 0.34\right)$ consistent with what is expected from the forward-shock mechanism.

On the other hand, considering the case of $\nu_{m}<\nu_{\mathrm{opt}}<\nu_{c}$, the decay phase of the light curve $\left(\alpha_{2}=-0.51 \pm 0.01\right)$ would give $p=1.68$ for the uniform ISM case and $p=1.01$ for the preburst wind case in the standard afterglow model. These values are smaller than the expected value $(p>2)$. Moreover, if we consider the afterglow with a flat-spectrum electron distribution $(1<p<2)$, the obtained value $p=0.74$ for the uniform ISM case and $p=-3.92$ for the preburst wind case are also inconsistent with the theoretical value (Dai \& Cheng

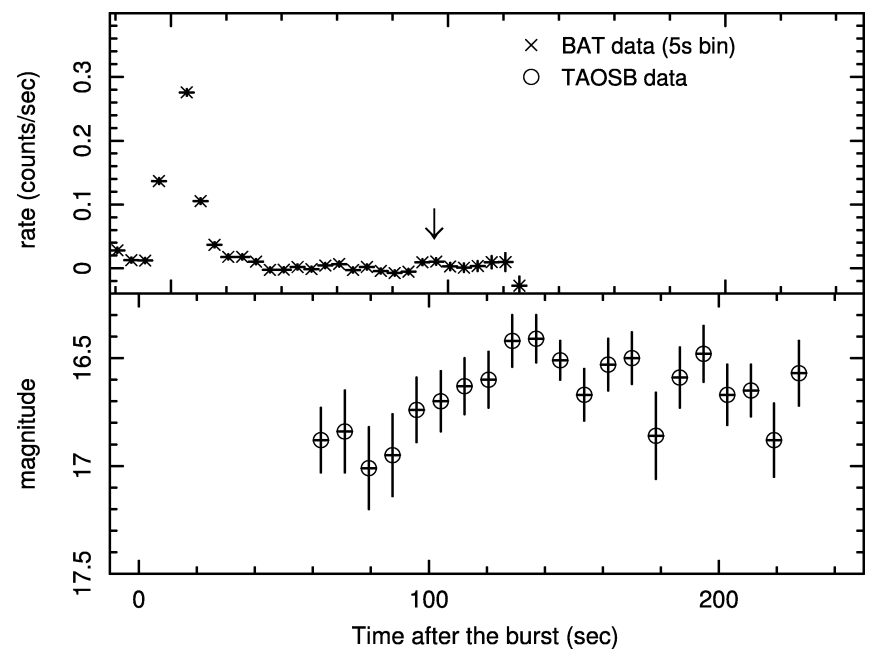

FIG. 4.- Swift 15-350 keV BAT light curve (5 s binning) and the TAOS$\mathrm{B}$ light curve (5 s sampling). The inserted arrow denotes the weak third peak of the BAT data. 
2001). The decay phase in GRB 071010B therefore cannot be well explained by the standard afterglow model. Without spectral or other multiband measurements in the early phase of GRB $071010 B$, it is not possible to verify whether the brightening was indeed caused by the synchrotron peak frequency, but even if it was, an additional mechanism is probably needed to account for the shallow decay after the brightening.

GRB 071010B was detected to have a jet break (Kann et al. 2007c; Im et al. 2007c; Y. Urata et al. 2008, in preparation), suggesting a viewing angle nearly along the jet axis. This excludes the possibility that a tail of prompt emission combined with an afterglow could produce a shallow decay when observing outside the edge of the jet (Eichler \& Granot 2006) in GRB 071010B.

The standard GRB afterglow model assumes an initially steady energy. The GRB is produced by the "internal shocks," i.e., a series of collisions between shells moving at different velocities. The energy hence changes when the slow material starts to be left behind. As the initially high-speed material is slowed down by the surrounding environment, the slower material eventually catches up and produces refreshed shocks. Significant continuous energy injection is possible and influences the fireball during the deceleration phase. The forward shocks will be refreshed and decelerate slower than in the standard scenario. This would change the decay slope of the light curve from the canonical one to a shallower one, as is evidenced in GRB 071010B.

Energy injection is expected if the energy distribution over the fireball surface is not uniform. The two-component jet and the patchy jet model have been proposed to explain the nonuniformity. The two-component jet model requires one component to be ultrarelativistic to power the GRB, whereas the other component is a moderately relativistic cocoon (Meśzaŕos \& Rees 1993; Berger et al. 2003; Zhang et al. 2004; Huang et al. 2004; Granot 2005). The afterglow of the cocoon contains more energy than the narrow component, thereby producing a noticeable "bump" in the light curve, instead of just changing the rate of the flux decay, but such a bump should be visible only after $10^{4} \mathrm{~s}$ after the burst (e.g., GRB 030329; Huang et al. 2006). It is obvious that the two-component jet model cannot explain the GRB 071010B light curve.

Alternatively, the light curve of GRB 071010B can be explained by the patchy jet model (Kumar \& Piran 2000), which assumes the initial fireball ejecta to have large energy fluctuations in angular direction. This results in jets with distributed values of the Lorentz factor $(\Gamma)$, so observers along different viewing angles would receive different fluxes. Furthermore, with diversified values of $1 / \Gamma$, emission from different patches becomes observable at different time, thus rendering a shallow afterglow light curve as detected in GRB 071010B.

In summary, the prompt optical emission for GRB 071010B was detected within 1 minute after the burst. No significant correlation with the prompt $\gamma$-ray emission was found. Our data of the first 3 minutes combined with measurements made by other groups within 2 days after the burst show the temporal evolution of GRB 071010B to consist of a slight brightening followed by a shallow decay. It is likely that the synchrotron peak frequency passed through the optical wavelengths and caused the early brightening, whereas the continuous injection by patchy jets was responsible for the slow decay that followed. The detection of GRB 071010B demonstrates the potential of the TAOS system to respond efficiently to a GRB alert to detect both prompt optical emission and early afterglow.

We acknowledge the use of public data from the Swift data archive and C. Markwardt for discussions. Work at NCU was supported by the grant NSC 96-2112-M-008-024-MY3. Work at the CfA was supported in part by the NSF under grant AST 05-01681 and by NASA under grant NNG04G113G. Work at ASIAA was supported in part by the thematic research program AS- 88-TP-A02 and Acdemia Sinica. Work at Yonsei was supported by Korea Astronomy and Space Science Institute. Work at LLNL was performed under the auspices of the US DOE in part under contract W-7405-Eng-48 and contract DE-AC5207NA27344. Work at SLAC was performed under US DOE contract DE-AC02-76SF00515.

\section{REFERENCES}

Akerlof, C., et al. 1999, Nature, 398, 400

Alcock, C., et al. 2003, Earth Moon Planets, 92, 459

Antonyuk, K., Rumyantsev, V., \& Pozanenko, A. 2007, GCN Circ. 7041

Berger, E., et al. 2003, Nature, 426, 154

Bertin, E., \& Arnouts, S. 1996, A\&AS, 117, 393

Beuermann, K., et al 1999, A\&A, 352, L26

Cenko, S. B., Cucchiara, A., Fox, D. B., Berger, E., \& Price, P. 2007, GCN Circ. 6888

Chen, W. P., et al. 2007, in IAU Symp. 236, Near Earth Objects, Our Celestial Neighbors: Opportunity and Risk, ed. G. B. Valsecchi \& D. Vokrouhlický (Cambridge: Cambridge Univ. Press), 65

Dai, Z. G., \& Cheng, K. S. 2001, ApJ, 558, L109

Eichler, D., \& Granot, J. 2006, ApJ, 641, L5

Gehrels, N., et al. 2004, ApJ, 611, 1005

Granot, J. 2005, ApJ, 631, 1022

Henden, A. 2007, GCN Circ. 6909

Huang, Y. F., Cheng, K. S., \& Gao, T. T. 2006, ApJ, 637, 873

Huang, Y. F., Wu, X. F., Dai, Z. G., Ma, H. T., \& Lu, T. 2004, ApJ, 605, 300

Im, M., Lee, I., \& Urata, Y. 2007a, GCN Circ. 6897

2007b, GCN Circ. 6920 2007c, GCN Circ. 6969

Kann, D. A., Hoegner, C., \& Filgas, F. 2007a, GCN Circ. 6884 2007b, GCN Circ. 6918

Kann, D. A., et al. 2007c, GCN Circ. 6935

Katz, J. I. 1994, ApJ, 422, 248

Kennea, J. A., et al. 2007, GCN Circ. 6878

Klunko, E., Marchenkov, A., Eselevich, M., Shulga, A., \& Pozanenko, A. 2007, GCN Circ. 6945
Kira, Y., et al. 2007, GCN Circ. 6931

Kocevski, D., Perley, D. A., Bloom, J. S., Modjaz, M., \& Poznanski, D. 2007, GCN Circ. 6919

Kumar, P., \& Piran, T. 2000, ApJ, 532, 286

Lehner, M. J., et al. 2008, PASP, in press (arXiv:0802.0303)

Markwardt, C. B., et al. 2007, GCN Rep. 92.1

Meśzáros, P. 2002, ARA\&A, 40, 137

Meśzaŕos, P., \& Rees, M. J. 1993, ApJ, 405, 278

Narayan, R., Paczyński, B., \& Piran, T. 1992, ApJ, 395, L83

Oksanen, A. 2007, GCN Circ. 6873

Rees, M. J., \& Meśzaŕos, P. 1992, MNRAS, 258, 41P 1994, ApJ, 430, L93

Rumyantsev, V., \& Pozanenko, A. 2007, GCN Circ. 6977

Rykoff, E. S., et al. 2005, ApJ, 631, L121

Saril, R., \& Piran, T. 1995, ApJ, 455, L143

1997a, ApJ, 485, 270

1997b, MNRAS, 287, 110

Stern, D., Perley, D. A., Reddy, N., Prochaska, J. X., Spinrad, H., \& Dickinson, M. 2007, GCN Circ. 6928

Stetson, P. B. 1987, PASP, 99, 191

Templeton, M., Kann, D. A., Oksanen, A., \& Henden, A. 2007, GCN Circ. 6903

Urata, Y., et al. 2007, ApJ, 655, L81

Vestrand, W. T., et al. 2005, Nature, 435, 178

- 2006, Nature, 442, 172

Xin, L. P. 2007, GCN Circ. 6924

Zhang, W., Woosley, S. E., \& Herger, A. 2004, ApJ, 608, 365 